\title{
Becoming a role model: the breastfeeding trajectory of Hong Kong women breastfeeding longer than 6 months
}

\section{Abstract}

While a substantial proportion of breastfeeding women stop early in the postpartum period, some women are able to breastfeed for longer periods. The aim of this research was to explore the experience of breastfeeding with a subsample of Hong Kong women who have breastfed for longer than 6 months. Participants $(n=17)$ were recruited from a larger infant-feeding study $(n=360)$ conducted in tertiary-care hospitals in Hong Kong. In-depth qualitative interviews were conducted and content analysis was used to analyse the data. Data analysis revealed four themes that encompassed the women's experiences: (1) making the decision, (2) maintaining family harmony, (3) overcoming barriers, and (4) sustaining lactation. Antenatally, participants anticipated that breastfeeding would be very 'difficult' and described how the practice did not fit with the image of a professional woman in Hong Kong. Despite family opposition, frequently from their mother-in-law, and lack of societal acceptance, difficulties were overcome by what the Chinese people call hung-sum or determination. This study highlights unique cultural and social findings affecting breastfeeding women in Hong Kong which may be useful to health-care providers working with Chinese women locally and internationally.

\section{Introduction}

Breastfeeding provides optimal and complete nutrition for newborn babies. The benefits of breastfeeding to both the infant and the mother have been widely recognized (World Health Organization, 2001). Despite these benefits, a substantial portion of women in developed countries still do not breastfeed. Furthermore, women who do choose to breastfeed often do so for only short periods. The health benefits of breastfeed-

\footnotetext{
*Corresponding author. Department of Nursing Studies,
} University of Hong Kong, 4/F, Academic and Administration Block, Faculty of Medicine, 21 Sasson Road, Hong Kong. Tel.: +852-2819-2643; fax: +852-2872-6079.

E-mail address: tarrantm@hku.hk (M. Tarrant). ing are dose-dependent, meaning that breastfeeding longer and exclusively confers greater health benefits to the infant (Lawrence, 1997). Exclusive breastfeeding for 6 months is recommended and results in reduced gastrointestinal morbidity for the infant while ensuring optimal growth and development (Kramer and Kakuma, 2003).

Women in Hong Kong, like women in many other industrialized countries, are increasingly choosing to breastfeed their infants. The latest data indicate that $60 \%$ of all new mothers now initiate breastfeeding, up from 19\% in 1981 (Khin et al., 2002). Despite recommendations from the World Health Organization (WHO) (World Health Organization, 2001) and the local Department of Health (Hong Kong Department of 
1 Health, 2003) for exclusive breastfeeding up to 6 months of age and continued breastfeeding up to 12 months of

3 age, few Hong Kong women exclusively breastfeed and most stop breastfeeding within the first few months

5 postpartum. Data on duration and exclusivity of breastfeeding in Hong Kong is sometimes conflicting,

7 reflecting the often fragmented nature of data collection. One population-based study of the 1997 birth cohort in

9 Hong Kong found that only $20 \%$ of infants were still breastfeeding at 1 month of age and just over $10 \%$ were

11 still breastfeeding at 3 months of age (Leung et al., 2002a, b). Another large breastfeeding survey showed

13 that from 1997 to $2000,35-39 \%$ of women breastfed for at least 1 month, $22-28 \%$ breastfed for 4 months, and

$1515-19 \%$ breastfed for at least 6 months (Khin et al., 2002). A longitudinal non-population based study found

17 that up to $20 \%$ of women continued to breastfeed for 6 months or longer (Dodgson et al., 2003a).

19 Although Khin et al. (2002) report that rates of exclusive breastfeeding at 1 month increased from $14 \%$

21 in 1997 to $22 \%$ in 2000 , Chan et al. (2000) found that $44 \%$ of breastfeeding participants $(n=44)$ who had

23 intended to exclusively breastfeed for 3 months or more, had completely weaned their infants by their sixth week

25 postpartum. Of the participants who continued to breastfeed past 3 months, $13 \%$ were supplementing

27 their breastfeeding with artificial milk (Chan et al., 2000). Overall, women who initiate breastfeeding in

29 Hong Kong are older, more highly educated, and less likely to return to full-time employment in the post31 partum period (Leung et al., 2002a, b). Furthermore, Hong Kong has one of the highest Cesarean Section

33 rates $(27.1 \%)$ in the world (Leung et al., 2001), a factor which has been demonstrated to adversely impact breastfeeding rates (Leung et al., 2002a, b).

Traditional Chinese postpartum practices and family

37 dynamics also play a substantial role in Hong Kong women's decisions about breastfeeding. Chinese

39 mothers often spend the first month after delivery convalescing at home, away from societal obligations

41 (Fok, 1996). This is referred to as doing-the-month or Chóh Yuht in Cantonese (the Hong Kong Chinese

43 dialect). During this time, traditional practices dictate that the new mother rest in bed and eat prescribed foods

45 that promote recovery from childbirth and increase milk production (Fok, 1996; Holroyd et al., 1997). Adherence

47 to these practices is considered preventive, as it is believed that it helps to restore health and prevent

49 debilitating diseases, such as asthma and arthritis, later in life (Pillsbury, 1982). This period can be isolating for

51 the woman as contact with the outside world is minimized so that the mother can rest and recover

53 (Chee and Horstmanshof, 1996).

In Confusion-based societies, breastfeeding practices

55 are also influenced by maternal position within the family and roles of other family members. The mother- in-law, traditionally the most powerful family member, exerts a special influence on infant feeding decisions. During interviews with more than 4000 Chinese women in Singapore over a 5-year period, Fok (1996) found that mothers who wanted to breastfeed would not do so if any significant other opposed their decision. This maternal behaviour was attributed to a characteristic Chinese desire to 'save face' (a complex etiquette for social interactions) and preserve family relationships by agreeing with those of a higher social status such as the mother-in-law. Furthermore, participants in studies of postnatal practices in both Hong Kong-born women and migrant Chinese women reported that they adhered to the traditional dietary and activity restrictions if encouraged to do so by their spouse or older relatives (Holroyd et al., 1997; Matthey et al., 2002).

In Hong Kong, $75 \%$ of women aged 25-45 years work full-time (Hong Kong Census and Statistics Department, 2001). The maximum maternity leave available is 10 weeks and a minimum of 2 weeks must be taken before the woman's expected date of confinement (Hong Kong Labour Relations Promotion Unit, 2002). Compliance with maternity leave regulations, however, varies with employers. Work environments rarely provide support for lactating women (Tarrant et al., 2002). Leung et al. (2002a, b) found that mothers who were employed full-time were $25 \%$ less likely to breastfeed. In another Hong Kong study, where 235 breastfeeding mothers were surveyed about their reasons for early weaning, $67 \%$ reported that their maternity leave was insufficient time to establish nursing (Chee and Horstmanshof, 1996).

Although the majority of breastfeeding women in Hong Kong wean early in the postpartum period, a small proportion do breastfeed for extended periods of time (Dodgson et al., 2003a). Exploring and describing the experiences of these women can provide valuable information to health professionals trying to improve breastfeeding rates among Chinese women both locally and internationally. Therefore, the specific objectives of this investigation were to: (a) explore the breastfeeding experiences of first-time mothers who had breastfed for 6 months or longer, and to (b) identify factors that contributed to their sustained breastfeeding.

\section{Research method}

\subsection{Study design}

The research reported in this paper was conducted as part of a larger longitudinal infant feeding study, which examined the relationship between motivational and situational variables and primapara mothers' infant feeding behaviours (Dodgson et al., 2003a, b; Tarrant et al., 2002). Data gathered for this investigation were
105 
1 collected using a qualitative descriptive design, which explored and described participants' experiences and

3 how these related to other social and cultural factors. This design is consistent with the study objectives

5 because it presents a comprehensive summary of people's experiences in everyday terms, and is the

7 preferred method when the aim of the researcher is to obtain answers to questions of special relevance to practitioners (Sandelowski, 2000).

Participants were recruited into the larger study during the immediate postpartum period from two public hospitals on the island of Hong Kong. In each hospital more than 3000 births occur per year, primarily low-risk deliveries. The following criteria were used for selection of study participants: (i) primaparas; (ii)

19 Cantonese speaking; (iii) Hong Kong residents for more than 1 year; and (iv) no serious medical or obstetrical

21 complication. Breastfeeding participants from the larger study were invited to take part in this investigation if they continued to breastfeed for 6 months or longer $(n=31)$. Twenty-four mothers were invited to participarticipation rate of $71 \%$. The remaining seven eligible participants were not approached to participate in the study as data saturation had been achieved. Two 29 participants interviewed for this study also participated in a previous qualitative investigation of the factors

31 affecting breastfeeding initiation carried out at 1-month postpartum (Tarrant et al., 2002).

\subsection{Data collection instrument}

In this investigation, person-centred in-depth interviews (Levy and Hollan, 1998) were carried out with the participants between 6 and 8 months postpartum. An interview guide with open-ended questions was developed for the study. Discussion topics for the interviews included the participants' decision-making process, their breastfeeding experiences, the feelings of their husband and family members about their breastfeeding decision, breastfeeding support, and their experiences sustaining breastfeeding while returning to work and resuming normal activities.

\subsection{Data collection procedures}

In-person interviews were conducted with participants by the first author and a translator over a 6-month period from May to November 2001. Interviews were conducted primarily in the participants' homes. The husbands of eight participants were present during the interviews and chose to participate to varying degrees. After informed consent was obtained, interviews were

conducted in either Cantonese $(n=7)$ or English $(n=10)$ and with the participants' permission were audio taped. A trained translator was used to interview all Cantonese-speaking participants and was present to assist with any required translation at all but five of the English interviews. In the five interviews where the translator was not present, participants were asked if they wished a translator to be present but in each case stated that they did not feel the need as they were bilingual. The interviews lasted from $45 \mathrm{~min}$ to $2 \mathrm{~h}$.

The seven Cantonese interviews were translated and transcribed into English by a bilingual translator/ transcriptionist. A bilingual research assistant reviewed the taped interviews and validated the accuracy of the transcription and translation of each interview. The transcriptionist transcribed the ten English interviews verbatim and the interviewer verified the accuracy of the transcription. After each interview, the researchers reviewed the transcripts to gain further insight into the mothers' experiences, which then provided information used to refine subsequent interview questions. In this way, questions raised during data collection were further explored in subsequent interviews increasing the richness of the data (Morse and Field, 1995).

\subsection{Data analysis}

The researchers reviewed all transcripts repeatedly and independently. Thematic content analysis was conducted, following the guidelines put forth by Morse and Field (1995). An inductive open coding procedure was employed whereby the data were coded and codes were grouped together and then categorized. Subsequent to reflection, themes emerged that captured a substantial portion of the coded data. Once the themes were identified, the transcripts were reviewed again to validate the thematic analysis and to ensure that all meaningful interview data had been analysed. After independent analysis, the researchers compared categories and themes and reviewed the data until a consensus was reached concerning the thematic structure. Throughout data analysis, the NVIVO software program (Richards, 1999) was used to assist with organization, management, and analysis of the data.

\subsection{Ethical considerations}

Prior to data collection, the Institutional Review Boards of the Faculty of Medicine, University of Hong Kong and the two participating hospitals, granted ethical approval. Informed written consent was obtained from each participant prior to data collection. Confidentiality was maintained throughout the research process by ensuring that participants' names were not associated in any way with the data collected and by keeping all research materials in a secure location. 


\section{ARTICLE IN PRESS}

\section{Study findings}

3

The demographic profile of the participants is presented in Table 1. The age of participants ranged 5 from 27 to 38 years. The mean age of 32 years $(\mathrm{SD}=3.73)$ was slightly older than the mean age of

730.5 years in the larger study sample (Dodgson et al., 2003b). All participants were married. Overall, partici-

9 pants exceeded their expected duration of breastfeeding by approximately 16 weeks and the majority $(n=14)$

11 were still breastfeeding at the time of data collection. Almost $65 \%(n=11)$ returned to work full-time after delivery with a mean return-to-work time of 9.8 weeks $(\mathrm{SD}=3.10)$.

Following thematic analysis (Morse and Field, 1995), four themes emerged that depicted the breastfeeding trajectory of the participants over the course of their first 6 months of breastfeeding: making the decision, main-

Table 1

Profile of participants

\section{Characteristic}

Age

Intention to breastfeed (weeks)

Actual duration of breastfeeding (weeks)
$32.06(3.73)$

$24.13(14.07)$

$39.82(11.90)$

$9.80(3.10)$
Table 2

The thematic structure of the trajectory of Hong Kong women who breastfed longer than 6 months

Making the decision

Societal Influences

Expectations of the breastfeeding experience

Maintaining family harmony

Doing-the-month (Chóh Yuht)

Family resistance

Burden of breastfeeding

Persistence in overcoming barriers

Seeking support

Anti-breastfeeding advice

Returning to work

Determination (Hung-sum)

Sustaining lactation

Achieving validation

Becoming a role model

breastfeed their babies. They don't think a professor or a doctor will breastfeed their babies, I don't know why. Maybe they have some prejudice because in Hong Kong we have many immigrants from China. They think that only those immigrant women will do that and Hong Kong women don't do that. I think it is a very, very bad mindset. But also in Hong Kong women are money-oriented. There is a belief that home-makers or house-wives do not have much social status. We call them 'Si Lai', do you know 'Si Lai'? The interpretation is of a layman, a lower class housewife. Because they do not earn income for the family they are treated as lower class. If I can earn tens of thousands of [Hong Kong] dollars a month they will think I am a high level professional career woman. (Participant \#2)

Participants agreed, however, that the general public's perceptions about breastfeeding are changing and breastfeeding is slowly becoming more acceptable within Hong Kong society.

\subsubsection{Expectations of the breastfeeding experience}

Breastfeeding, like other social behaviour, is often strongly influenced by one's family and peers. Although all participants breastfed for at least 6 months and some planned it this way, not all initially anticipated continuing to breastfeed for as long as they did. Because of discussions with family members and friends, most women held the expectation that breastfeeding would be a difficult process, and only a few were actually advised to breastfeed. Therefore, most participants began breastfeeding thinking that they would 'try it' and did 
1 not plan specifically how long they would continue to breastfeed.

At that time, I did not plan how long I would

breastfeed him because I did not know if I could breastfeed. Because some of my friends told me that it had not been a good experience for them breastfeeding their babies, I just wanted to try my best.

\section{(Participant \#12)}

All participants knew other mothers who had tried to breastfeed but had failed or had given up very early in the postpartum period. As one participant stated, many of her peers expected her to have a similar result:

My friends didn't advise me not to breastfeed the baby, they just said it's not easy. It's not easy and it is very difficult they said. It is difficult... at the beginning they said. They thought I would give up in the first month because they said it is not easy. (Participant \#2)

Participants reported, however, that as their breastfeeding experience progressed their confidence grew and they were able to continue for longer than expected.

\subsection{Maintaining family harmony}

In Chinese culture, maintaining family harmony by respecting the wishes of one's elders is expected of all young adults. During the postpartum period, this expectation became problematic for many participants as the advice and guidance of their elders often conflicted with their own desires. Participants' descriptions of their attempts to maintain family harmony are described under three thematic subcategories: doing-themonth (chóh yuht), family resistance to breastfeeding, and the burden of breastfeeding.

\subsubsection{Doing-the-month (chóh yuht)}

In traditional Chinese society, the postpartum period is replete with rituals that new mothers are expected to follow while doing-the-month (Chóh Yuht). To please their elders and to minimize family conflict, all but two study participants reported following at least some of the traditional practices prescribed during the immediate postpartum period. Most, however, reported that they did not believe there were any specific benefits to following these practices. Since they 'did no harm' participants followed them to maintain family harmony and to please their mother or mother-in-law.

Participants primarily followed the dietary prescriptions and reported most difficulty complying with the restrictions on bathing, showering, and washing their hair. Foods such as chicken, eggs, ginger, and fish and papaya soup were consumed regularly to help promote healing, to increase the dissolving of postpartum blood clots, and to increase the milk supply. One participant described her experience:

Actually I didn't want to follow it, but I had to. Because my mom and mother-in-law asked me to follow this culture. But I don't mind. The horrible thing is that you can't go outside and you can't wash your hair.... But, I don't want to see anyone unhappy. So I think that only one month is fine. But, I said only one month. I can't continue to do something that horrible. (Participant \#10)

Three participants even reported bathing and washing their hair 'in secret' so that their mother or mother-inlaw would not find out about it.

\subsubsection{Family resistance to breastfeeding}

Participants also described the strong opposition they frequently encountered from their family members over their decision to breastfeed their baby. Many of their family members associated formula fed 'chubby babies' with healthy babies. As their breastfed babies tended to be leaner, participants often met with family disapproval over their decision to breastfeed. A number of these participants felt a generation or a gender gap existed in understanding the value of breastfeeding and tried to help other family members understand why it was important to provide their own milk to their baby. One participant summarized it this way:

Some women's mothers or mothers-in-law, don't want them to breastfeed their babies because they think the formula is better than their own milk and they discourage their daughter or daughter-in-law. I think for the older generation, for example, over 60 years old, they recommend breastfeeding but for those in their $40 \mathrm{~s}$ or $50 \mathrm{~s}$, they don't recommend it. They think the formula is the best. I think for those who are over 60 , they didn't have formula at that time, they only had breastfeeding. I think the commercials also give them the wrong concept that formula is the best. (Participant \#17)

As maintaining family harmony was a priority, participants were expected to defer to the wishes of her in-laws, even by her own family. One participant described her anguish in trying to continue breastfeeding against strong family opposition:

My family and all my relatives do not support me on breastfeeding.... I was always feeling very unsupported because my husband pressed me to give formula milk to the baby.... Initially, my husband didn't object but when the baby's weight dropped to a little over 5 pounds. She had jaundice and the light but the results were not good. Some people told him that if you use formula milk, you won't have this 
problem of jaundice. So my husband wanted me to use formula milk and then it got to be more and more. My baby was hospitalized twice. The first time, she almost needed a blood transfusion. [My husband] just wanted the best for the baby so he wanted me to feed formula milk. I know that breast milk is the best milk because it has materials that can build up the baby, the brain, so I must give her the breast milk. The second time she was in the hospital, I thought she may die. I was afraid so I made a choice to tell the doctor 'I won't give her breast milk'. My relatives didn't support me (sobbing). My own mother comes from a very traditional family. My mom wanted me to obey my husband's family and my husband's father and mother didn't want me to breastfeed.... After my mother found out the condition of the baby, she repeatedly called me up, my sisters too, telling me to stop breastfeeding, in a scolding manner. They questioned me as to why I kept doing this? I talked and explained to my mother openly about this. But I did not say a word to my parents-inlaw because I don't want to irritate them and to cause the relationship to go sour. So I just restrained myself on this matter. (Participant \#9)

Another participant described her experience:

My husband complains to me, he feels the baby is not so big as other babies, he is still very small, still very hungry. 'Maybe your breast feeding is not so successful or your milk supply is too little for the baby.' Every day, he pushes me to stop. I feel very depressed and I can say once or twice I cried over this. Because how come I want to give the best present to my baby but they feel that it's not good for my baby.... My husband always pushes me to stop and also his family. When sometimes I go to work, they use my pumped milk to feed my baby, but sometimes when the baby is nearly finish the bottle they will try to add the instant milk to the baby.... I feel they are not so supportive of me. (Participant \#1)

\subsubsection{The burden of breastfeeding}

As part of doing-the-month, the 1-month period of postnatal rest is seen as very important and failure to comply will result in long-term health consequences for the new mother (Pillsbury, 1982). While some participants reported that their family members knew breastfeeding was best for the baby and supported their decision to breastfeed, family members also frequently expressed concern about the 'burden' of breastfeeding for the new mother. Participants' families expressed concern that breastfeeding is so demanding and exhausting for her that she will be unable to get the required amount of rest. These worries appeared to be expressed more often by family members who had not previously breastfed.

My mother and auntie, at the beginning, they perceived it was tough for me to breastfeed. I did not sleep well... they wondered if I was exhausted.... The main reason is that they thought it was tough for me. They were afraid. Three days after being discharged from the hospital, I had a relatively severe flu. Later, they told me not to breastfeed because I would get worse. In the Chinese culture, it is difficult to recover when a woman is ill within one month of giving birth.... Also, at the beginning, my mother-in-law told me not to breastfeed because she thought it would be tough for me. She wondered if I could manage. (Participant \#11)

Mothers or mothers-in-law who had breastfed their own children were less likely to advise participants to discontinue breastfeeding. Instead of encouraging mothers to supplement or switch to infant formula, family members who had personal breastfeeding experience would provide other supportive help (e.g., house cleaning and cooking) so that the new mother could concentrate completely on breastfeeding and not be overwhelmed with other tasks.

My mother is very supportive because my mother is the old generation type, she breastfed all of us. My mother has four children, all breastfed. She knows that it is good, of course it is good. So my mother has always supported me. (Participant \#6)

Other roles within the family can be affected by the choice to breastfeed. Family members, especially the mother-in-law, often monitor the new mother's compliance with traditional postpartum practices. Two participants speculated that when a new mother breastfeeds, her mother-in-law may feel that she is not fulfilling her role as caretaker of the mother and baby during the postpartum period, as she would be if the baby was being formula fed.

\subsection{Persistence in overcoming barriers}

Continuing to breastfeed beyond 6 months required persistence by the participants to overcome the barriers they faced. This process of overcoming barriers is further described under the following thematic subcategories: seeking support, anti-breastfeeding advice, returning to work, and determination (hung sum).

\subsubsection{Seeking support}

Participants who did not have family support or who felt they needed extra help often sought support from breastfeeding professionals and peer support organizations in Hong Kong. Participants contacted lactation 
1 consultants, nurses in the government-run Maternal and Child Health Centres, various breastfeeding hotlines,

3 and the Hong Kong Breastfeeding Mothers Association. A few sought advice from friends and peers who had 5 breastfed. Three women who had live-in domestic helpers, reported that these women were a valuable 7 source of support as they had previous breastfeeding experience. The following participant underscored the importance of support to the new mother:

Fortunately, our domestic helper, she had experience with successful breastfeeding and she taught me how to do it when I was in the hospital. The support from the surrounding people is very important because if at that point, if anybody told me to stop, I might have stopped. But fortunately, they kept on telling me to try and they told me that it is the best for your son, so please do it. And so I can persist with it. (Participant \#11)

\subsubsection{Anti-breastfeeding advice}

Unfortunately, family members were not the only people to recommend that new mothers discontinue breastfeeding. Twelve out of the 17 participants reported that a health professional, in most cases a General Practitioner, had advised them to completely discontinue breastfeeding. These recommendations were given for a variety of inappropriate reasons, including atopy and maternal illness, or simply because they considered it no longer necessary for the participant to breastfeed. Participants often reported that General Practitioners told them that breastfeeding past 2-3 months was unnecessary and offered no extra benefits for the baby. As some of the participants identified, they themselves often had a greater understanding of breastfeeding than the professionals from whom they sought advice.

When my son was four months old, I had a very severe cold. I went to my family doctor and I asked him 'can I still breastfeed when I take the medicine?' And he asked how old my baby was and I said four months. He said, 'oh, it's a great opportunity to quit. You do not need to breastfeed him anymore. He has gotten all of the benefits from you; you can now change to formula.' I was very very disappointed as before that I thought he was a very professional doctor but after that event, I changed my opinion (laughing). (Participant \#17)

Participants also discussed their in-hospital experiences while initiating breastfeeding. Participants, who were sure of their decision to breastfeed and insisted that their babies have only breast milk, reported that nurses and hospital staff were supportive and did not encourage formula use. Participants who were less confident and who experienced difficulties initiating breastfeeding, however, often reported that hospital nurses encouraged supplementation. One participant described her experience of initiating breastfeeding while her baby was being investigated for a cardiac anomaly:

Often times the hospital procedures make it very difficult when there is something, you know, if there's maybe something wrong with the baby or they are checking to see if there is something wrong with the baby. The hospital procedures make it very difficult for the mother to keep breastfeeding the baby. And also, some of the nurses, although they do not verbally advise you to stop breastfeeding because the baby needs to stay in the hospital, but their actions made me feel that they actually didn't like us to do the breastfeeding. Some of them are this way but not all. I think they think it is easier for them to do their work if I do not breastfeed. (Participant \#15)

Another participant related her experience of trying to breastfeed her baby immediately after birth.

Right after I gave birth the baby was taken away immediately because of hypothermia. I think she was a Nursing Officer, she said that you can feed her anything, she will grow. I felt that was discouraging. (Participant \#16)

Participants also stressed that while nurses were helpful and tried to support them as much as possible, nurses were often too overburdened to be able to offer much individualized breastfeeding support.

\subsubsection{Returning to work}

Although maternity leave in Hong Kong is short, eleven participants were able to balance a return to fulltime employment and the continuation of breastfeeding. While most participants would have liked a longer period of time at home with their babies, for financial reasons they had to return to work full-time. Expressing their breast milk with a pump was the strategy used by all full-time workers to enable them to continue breastfeeding while working. Only two women had a designated nursing mother's room in their workplace. Few participants had separate rooms or places that were sufficiently private and comfortable for pumping. The toilet, while not optimal, was the place most frequently used by the participants to express their milk. Adequate preparation was identified as being important in allowing participants to continue breastfeeding while working. Having discussions with supervisors, ensuring there was somewhere to store the milk, and starting to pump before returning to work were strategies that various participants used to smooth this transition.

\subsubsection{Determination (Hung-sum)}

Although participants expected breastfeeding to be 'difficult', most still found the initial experience some- 
1 what overwhelming. All but one participant reported insufficient milk in the first days and weeks postpartum.

3 Many experienced pain and discomfort from cracked and sore nipples while trying to position the baby 5 correctly. Two participants experienced mastitis. All experienced fatigue and sleep deprivation. According to

7 some participants, it is necessary for both mother and baby to acquire experience and skill in breastfeeding to

9 be successful. They stressed that both parties require practice and need to become acquainted with each other

11 before competence can be achieved. While participants described often wanting to quit breastfeeding, they

13 identified having what Chinese people call hung-sum or determination enabled them to overcome the difficulties they encountered:

In the beginning, I was not accustomed to it. For example, the frequency of feeding, sometimes it's hard to get a good night's sleep. But you must be determined to solve these problems. You must be patient or else you will give up easily. (Participant \#3)

Another participant discussed how she believed that women who quit breastfeeding simply lacked determination:

I've got a friend who just gave birth to a baby two days after me, she can't breastfeed. And I know, from relatives and friends, only half of them can breastfeed. Almost half cannot do it and even those half who do breastfeed, they quit when they go back to work. For those who can't breastfeed, they always blame it on something: 'there isn't enough [milk] for the baby; he/she cries a lot and that makes them worried and upset; the breastfeeding is hurting her' those kind of excuses. They don't persist so maybe their determination is not that high. (Participant \#14)

\subsection{Sustaining lactation}

After overcoming breastfeeding barriers, participants described the sense of accomplishment they received from their breastfeeding experience. They were comfortable in their role and subsequently were able to promote breastfeeding to their pregnant friends and family members. Participants' experiences are described under two thematic subcategories: achieving validation and becoming a role model.

\subsubsection{Achieving validation}

Almost all participants identified a point at which they felt they achieved a 'breakthrough' and sustaining lactation became easy and natural. Depending on the participant, this breakthrough point occurred anywhere from 2 weeks to 2 months. By 2 months, however, all participants had overcome the major barriers and were sufficiently comfortable with breastfeeding. As a result,

breastfeeding their first child was rewarding and validating experience for all mothers in this study. Participants perceived that their babies were much healthier and were developmentally and intellectually superior to the formula-fed infants of their peers and relatives. These perceptions validated the participants' breastfeeding decision. Participants viewed breastfeeding as the parents' contribution to their child's future and they felt great pride in this achievement. One father described the couple's feelings as parents:

We find there is a difference between our son and other boys. For example, he is very healthy and strong. So far, he hasn't needed any medical consultations, none. Instead ... my brother's son, he has one every month, it is very regular. His son needs to go to the doctor but our son is very strong, even in this season.... So we think that breastfeeding builds a good foundation for all kids, especially for our kids. (Husband of Participant \#17)

Another mother stated:

In the ward in the opposite bed, that baby is one day older than my son. I breastfed my son but that mother did not. And now we are good friends, so I always compare the both of us. I always compare our sons and my son is stronger and... with our baby the muscles and bones are stronger. Her son is weaker. (Participant \#8)

Participants also reported validation from the changes in her family members' opinions about breastfeeding, as their babies grew and developed. Their strong and healthy babies demonstrated the superiority of breast milk over infant formula to family members who were previously sceptical of the benefits of breast milk. Although they may have initially encouraged formula feeding, often the family members' opinions about breastfeeding were transformed and they now supported the mother's decision.

\subsubsection{Becoming a role model}

Participants also viewed their breastfeeding experience as an opportunity to promote breastfeeding to their peers and family members. Although they did not push others to breastfeed, they were empowered by their experience and wanted to share it with others. Participants also acted as role models to encourage other pregnant women to initiate breastfeeding. Two participants described how their healthy babies encouraged those around them to breastfeed:

I have a sister-in-law who just had a baby. The first baby she had she didn't do any breastfeeding, she didn't even think about that. Now she can see my baby is big, healthy, and doesn't need to go see the doctor for a year.... And my sister-in-law can see the 
benefits of it and now she is trying breastfeeding. (Participant \#15)

I have a friend who will give birth to a baby in September. She decided to breastfeed the baby. They

\section{Discussion}

With few exceptions (Tarrant et al., 2002), the breastfeeding experiences of women in Hong Kong have not been extensively investigated. Because breastfeeding is a culturally laden activity (Stuart-Macadam and Dettwyler, 1995) one can not design promotion and support programs without taking cultural context into consideration. The cultural context of the breastfeeding experiences of women, who successfully breastfed for at least 6 months, is evident throughout the findings of this study. Additionally, insights into these experiences and suggestions for health professionals working with Chinese postpartum women in Asia and internationally to improve services are presented.

\subsection{Making the decision}

Study participants, all of whom were successful in breastfeeding, often expected the experience to be difficult. Similar to the findings of other researchers (Dodgson et al., 2002), this perception came from stories participants had heard about other women's breastfeeding experiences. These perceptions can discourage breastfeeding women and cause insecurity about their ability to successfully breastfeed. While women in this study were able to overcome their initial uncertainties and establish successful breastfeeding, the anticipation of breastfeeding difficulties may influence other mothers to choose infant formula over breastfeeding, contributing to the low initiation rates in Hong Kong. An association between breastfeeding intention and duration has been clearly identified (Dodgson et al., 2003b; Donath et al., 2003; Duckett et al., 1998; Lawson and Tulloch, 1995), suggesting that despite the success of participants in this study, if the majority of women do not intend to breastfeed for long periods of time, they are unlikely to do so. While some early breastfeeding difficulties are possible, women need to be aware that adequate education and lactation support in the early postpartum period can overcome these difficulties (Dennis, 2002).

The increased prosperity that Hong Kong has experienced over the past 20 years has lead many people to associate infant formula with affluence. Furthermore, because many immigrant women breastfeed, Hong

Kong women may perceive that only poor migrant women breastfeed. In this respect, breastfeeding is still largely tied to social class in Hong Kong as working women are given much more status and mothering seen as secondary (Martin, 1997). While the association between breastfeeding and low social class is a common myth, not only in Hong Kong, but in other developed countries, the opposite demographic effect has occurred in recent years. Research has consistently shown a strong correlation between breastfeeding and higher social class or indicators of social class such as education and income (Bourgoin et al., 1997; Leung et al., 2002a, b; Scott et al., 1999). With centralized health care and health promotion programs, agencies of the Hong Kong Government are in an ideal position to focus on changing societal misperceptions of breastfeeding. Public breastfeeding education campaigns specifically aimed at groups with lower breastfeeding rates (i.e., younger women with lower education levels) can be used to dispel these myths and increase breastfeeding initiation rates (Dennis, 2002).

The economic realities of life in Hong Kong often dictate that both parents work. As some participants have highlighted, however, many in Hong Kong society value women more for their financial role in the family, than for their role as a mother. While seemingly a consequence of the economic transition that has occurred in Hong Kong over the past $30-40$ years, this perception may also reflect the traditional ideals of Confucianism, often still at the root of current Chinese thinking (Chen, 2001; Cheng, 1990). Under Confucian thought, financial success and high societal status are perceived to offer advantages to the family unit and help to increase the chance that the family line will be continued (Nicol, 2003).

\subsection{Maintaining family harmony}

Confucian thought also emphasizes that a person is highly regarded for their ability to maintain harmonious relationships with others, especially family members (Tang, 1992). Consequently, it is common for Asian women to make their infant feeding decisions based on the wishes of significant others such as the mother, mother-in-law, and husband (Rossiter, 1998). Study participants often faced immense pressure from family members to discontinue breastfeeding or to supplement with infant formula. Although most were able to uphold their breastfeeding decision, they were nonetheless torn between their conviction that breastfeeding was best for their baby and the expectation that they should comply with their family's wishes. An antenatal assessment of the compatibility of breastfeeding with the family's values and beliefs about breastfeeding may help to identify those at increased risk for breastfeeding failure (Leff et al., 1994). Furthermore, focussing on how 


\section{ARTICLE IN PRESS}

1 family members can support the breastfeeding mother in ways other than formula supplementation is needed 3 (Dykes and Griffiths, 1998).

A key issue identified by study participants was that 5 family members perceived breastfeeding as placing a burden on the new mother and interfering with a

7 woman's ability to get the required rest in the postnatal period, possibly resulting in long-term health conse-

9 quences. In an increasingly Westernized Hong Kong society, fewer women strictly adhere to the traditional

11 Chinese postpartum practices. Consequently, family friction often results when the postpartum woman's

13 mother or mother-in-law holds traditional expectations. The new mother must balance her own beliefs and desires with the expectations of her elder family members.

17 Because positive family support is critical in maintaining lactation, significant family members need to be

19 included in childbirth and breastfeeding education programs. Educating family members on the physiolo-

21 gical benefits of breastfeeding, to not only the baby but the mother, may help mitigate the perception of

23 breastfeeding as a detrimental health practice. As the postpartum period is not only about the new mother fulfilling a prescribed role, breastfeeding can diminish the traditional role of the mother-in-law and may cause them to encourage formula feeding during this period.

29 Culturally congruent family based antenatal and postnatal education is desirable to overcome the cultural impediments that often hinder successful breastfeeding.

\subsection{Persistence in overcoming barriers and sustaining lactation}

Unfortunately, the problem of physicians advising clients to terminate breastfeeding for invalid reasons is not new and has been documented in various groups of breastfeeding women. Rossiter (1998) reported similar findings in Vietnamese women in Sydney and Dodgson et al. (2002) found that when American Ojibwe women experienced breastfeeding problems, physicians frequently recommended supplementing with or switching to infant formula. Abel et al. (2001) also identified inadequate and conflicting advice from health professionals as a primary reason for supplementary feeding and early cessation of breastfeeding. In a survey of practising physicians, only $64 \%$ knew that supplementing in the immediate postpartum period might contribute to breastfeeding failure (Freed et al., 1995). In a previous Hong Kong study, Hung et al. (1985) found that physicians' attitudes to breastfeeding were vague and inconsistent. Nurses have also been identified as a source of inconsistent advice about breastfeeding, promoting breastfeeding verbally while their actions discourage the practice (Hong et al., 2003, Tarrant et al., 2002).

Because of their position and status in Hong Kong society, physicians and other health professionals are held in high regard. While participants in this study largely discounted recommendations to discontinue breastfeeding made by physicians, many other mothers may not. General Practitioners are often the first person a new mother will turn to for advice and guidance if she is experiencing problems in the postpartum period. To be able to offer the most appropriate guidance to their clients, it is essential that all health professionals acquire the latest information and knowledge about breastfeeding. Health professionals have a responsibility to ensure that the breastfeeding experience is a positive one for new mothers and that it enhances women's adjustment to their new mothering role. Promoting breastfeeding as a practice that strengthens women, children, and their families will help to reduce the barriers that Hong Kong women currently face.

Study participants' experiences demonstrated that women can succeed at breastfeeding despite encountering many obstacles along the way. One factor identified by these women as a reason for their success was hungsum or their determination. Not all postnatal women however, have this same level of fortitude. While all women are physiologically capable of breastfeeding, it is a skill that requires practice by both mother and baby. Determination to succeed at breastfeeding is a complex phenomenon that requires both persistence and commitment by the mother (Bottorff, 1990). Antenatal education alone is insufficient to prepare new mothers for the breastfeeding experience (Ho and Holroyd, 2002). Confidence and competency in breastfeeding are gained through apprenticeship rather than books and didactic education sessions (Hoddinott and Pill, 1999). New mothers need exposure to positive role models who can provide support and counselling, especially in the early postpartum period. Peer support programs have been successful at providing these role models in other settings (Raisler, 2000; Schafer et al., 1998). Although there are two active mother-to-mother support organizations in Hong Kong, a new mother needs to have information on how to access these resources and be willing to do so.

\section{Study limitations}

This study relates the breastfeeding experiences of 17 middle-class women. The data presented reflects only the experiences of a small subgroup of the breastfeeding population. The purposive sampling strategy and the small sample size limit the generalizability of these findings. Further research is required to adequately
107

109 
1 represent the breadth and depth of experience of breastfeeding women in Hong Kong.

Communication was affected during some interviews as the interviewer only spoke English and several of the participants only spoke Cantonese. The interviewer relied on a translator to provide an accurate translation of the participants' dialogue. It is possible that some errors and misunderstandings occurred during interpretation, which could have resulted in misinterpretation of the words spoken by the participants. However, the

researchers believe that this problem was minimal as trained translators and transcribers were used and an external reviewer validated the accuracy of the transcriptions.

\section{Conclusion}

The findings of this investigation suggest a number of commonalities between breastfeeding women in Hong Kong who are successful at maintaining breastfeeding over time and women who live in other areas of the world, which previously have not been documented. In addition, this study begins to explore the cultural context in which breastfeeding women in Hong Kong negotiate their infant feeding practices, including family, health care and social norms.

\section{Acknowledgements}

This research was funded by the Research Services Section, University of Hong Kong and by the Department of Nursing Studies, University of Hong Kong.

\section{References}

Abel, S., Park, J., Tipene-Leach, D., Finau, S., Lennan, M., 2001. Infant care practices in New Zealand: a cross cultural qualitative study. Social Science and Medicine 53, 11351148.

Bottorff, J.L., 1990. Persistence in breastfeeding: a phenomenological investigation. Journal of Advanced Nursing 15, 201-209.

Bourgoin, G.L., Lahaie, N.R., Rheaume, B.A., Berger, M.G., Dovigi, C.V., Picard, L.M., Sahai, V.F., 1997. Factors influencing the duration of breastfeeding in the Sudbury Region. Canadian Journal of Public Health 88 (4), 238-241.

Chan, S.M., Nelson, E.A.S., Leung, S.S.F., Li, C.Y., 2000. Breastfeeding failure in a longitudinal post-partum maternal nutrition study in Hong Kong. Journal of Paediatrics and Child Health 36, 466-471.

Chee, Y.O., Horstmanshof, L., 1996. A review of breastfeeding practices in Hong Kong 1994/1995. Breastfeeding Review 4 (1), 7-12.
Chen, Y.C., 2001. Chinese values, health and nursing. Journal of Advanced Nursing 36 (2), 270-273.

Cheng, S.K., 1990. Understanding the culture and behaviour of East Asians: a Confucian perspective. Australian and New Zealand Journal of Psychiatry 24 (4), 510-515.

Dennis, C.L., 2002. Breastfeeding initiation and duration: a 1990-2000 literature review. JOGNN 31 (1), 12-32.

Dodgson, J.E., Duckett, L., Garwick, A., Graham, B.L., 2002. An ecological perspective of breastfeeding within a indigenous community. Journal of Nursing Scholarship 34 (3), 235241.

Dodgson, J.E., Tarrant, M., Fong, D.Y.T., Peng, X.H., Hui Choi, E.W.H., 2003a. Breastfeeding patterns of primiparous mothers in Hong Kong. Birth: Issues in Perinatal Care 30 (3), 195-202.

Dodgson, J.E., Henly, S.J., Duckett, L., Tarrant, M., 2003 b. Theory of Planned Behavior-based models for breastfeeding duration among Hong Kong mothers. Nursing Research 52 (3), 148-158.

Donath, S.M., Amir, L.H., Team, A.S., 2003. Relationship between prenatal infant feeding intention and initiation and duration of breastfeeding: a cohort study. Acta Paediatrica 92 (3), 352-356.

Duckett, L., Henly, S., Avery, M., Potter, S., Hills-Bonczyk, S., Hulden, R., Savik, K., 1998. A Theory of Planned Behavior-based structural model for breast-feeding. Nursing Research 47 (6), 325-336.

Dykes, F., Griffiths, H., 1998. Societal influences upon continuation of breastfeeding. British Journal of Midwifery 6 (2), 76-80.

Fok, D., 1996. Cross cultural practice and its influence on breastfeeding - the Chinese culture. Breastfeeding Review 4 (1), 13-18.

Freed, G.L., Clark, S.J., Lohr, J.A., Sorenson, J.R., 1995. Pediatrician involvement in breast-feeding promotion: a national study of residents and practitioners. Pediatrics 96 (3), 490-494.

Ho, I., Holroyd, E., 2002. Chinese women's perceptions of the effectiveness of antenatal education in the preparation for motherhood. Journal of Advanced Nursing 38 (1), 74-85.

Hoddinott, P., Pill, R., 1999. Qualitative study of decisions about infant feeding among women in east end of London. British Medical Journal 318 (7175), 30-34.

Holroyd, E., Fung, K.K.L., Lam, C.S., Sin, H.W., 1997. Doing the month: an exploration of postpartum practices in Chinese women. Health Care for Women International 13, 301-313.

Hong Kong Census and Statistics Department, 2001. Women and Men in Hong Kong: Key Statistics. Author, Hong Kong.

Hong Kong Department of Health, 2003. Breastfeeding policy. Department of Health, Hong Kong.

Hong Kong Labour Relations Promotion Unit, 2002. Maternity protection. The Government of the Hong Kong SAR Labour Department.

Hong, T.M., Callister, L.C., Schwartz, R., 2003. First-time mothers' views of breastfeeding support from nurses. MCN, American Journal of Maternal Child Nursing 28 (1), 10-15.

Hung, B.K.M., Ling, L., Ong, S.G., 1985. Sources of influence on infant feeding practices in Hong Kong. Social Science and Medicine 20, 1143-1150. 


\section{ARTICLE IN PRESS}

12

1 Khin, P.P., Cheung, S.L., Loh, T., 2002. Support and promotion of breastfeeding. Where are we now? Public Health and Epidemiology Bulletin 11 (3), 25-32.

Kramer, M.S., Kakuma, R., 2003. Optimal duration of exclusive breastfeeding (Cochrane Review). In: The Cochrane Library, Issue 4. Wiley, Chichester, UK.

Lawrence, R.A., 1997. A Review of the Medical Benefits and Contraindications to Breastfeeding in the United States. Maternal Child Health Bureau, Arlington, VA.

Lawson, K., Tulloch, M.I., 1995. Breastfeeding duration: prenatal intentions and postnatal practices. Journal of Advanced Nursing 22 (5), 841-849.

Leff, E.W., Gange, M.P., Jefferis, S.C., 1994. Maternal perceptions of successful breastfeeding. Journal of Human Lactation 10 (2), 99-104.

Leung, G.M., Lam, T., Thach, T.Q., Wan, S., Ho, L., 2001. Rates of cesarean births in Hong Kong: 1987-1999. Birth 28 (3), 166-172.

Leung, G.M., Ho, L., Lam, T., 2002a. Breastfeeding rates in Hong Kong: a comparison of the 1987 and 1997 birth cohorts. Birth 29 (3), 162-168.

Leung, G.M., Lam, T., Ho, L., 2002b. Breast-feeding and its relation to smoking and mode of delivery. Obstetrics and Gynecology 99 (5 part 1), 785-794. women and relation to postpartum mood. Health Care for
Morse, J.M., Field, P.A., 1995. Qualitative research methods for health professionals. Sage, London.

Nicol, J., 2003. Confucianism - the Root of Hong Kong's Search for Wealth. South China Morning Post, Hong Kong, p. A13.

Pillsbury, B.L.K., 1982. Doing the month: confinement and convalescence of Chinese women after childbirth. In: Kay, M.A. (Ed.), Anthropology and Human Birth. F.A. Davis, Philadelphia, PA.

Raisler, J., 2000. Against the odds: breastfeeding experiences of low income mothers. Journal of Midwifery \& Women's Health 45 (3), 253-263.

Richards, L., 1999. Using NVivo in Qualitative Research. Sage, Thousand Oaks, CA.

Rossiter, J.C., 1998. Promoting breast feeding: The perceptions of vietnamese mothers in Sydney, Australia. Journal of Advanced Nursing 28 (3), 598-605.

Sandelowski, M., 2000. Whatever happened to qualitative description? Research in Nursing \& Health 23, 334-340.

Schafer, E., Vogel, M.K., Viegas, S., Hausafus, C., 1998. Volunteer peer counselors increase breastfeeding duration among rural low-income women. Birth 25 (2), 101-106.

Scott, J., Aitkin, I., Binns, C., Aroni, R., 1999. Factors associated with the duration of breastfeeding amongst women in Perth, Australia. Acta Paediatrica 88, 416-421.

Stuart-Macadam, P., Dettwyler, K.A., 1995. Breastfeeding: Biocultural Perspectives. Adine De Guyter, New York.

Tang, N.M., 1992. Some psychoanalytic implications of Chinese philosophy and child-rearing practices. Psychoanalytic Study of the Child 47, 371-389.

Tarrant, M., Dodgson, J.E., Tsang, S.F., 2002. Initiating and sustaining breastfeeding in Hong Kong: contextual influences on new mothers' experiences. Nursing and Health Sciences 4 (4), 189-199.

World Health Organization, 2001. Global strategy for infant and young child feeding. World Health Organization, Geneva, pp. 1-5. Women International 23 (6-7), 567-575. 